\title{
Spreading of Powders in Powder Bed Additive Manufacturing: an Experimental Approach
}

\author{
Valerio Lampitella, Marco Trofa, Antonello Astarita and Gaetano D’Avino
}

Valerio Lampitella. Department of Chemical, Materials and Industrial Production Engineering, University of Naples Federico II, Piazzale Tecchio 80, Naples, Italy

Corresponding author: valerio.lampitella@unina.it

Marco Trofa. Department of Chemical, Materials and Industrial Production Engineering, University of Naples Federico II, Piazzale Tecchio 80, Naples, Italy

Antonello Astarita. Department of Chemical, Materials and Industrial Production Engineering, University of Naples Federico II, Piazzale Tecchio 80, Naples, Italy

Gaetano D’Avino. Department of Chemical, Materials and Industrial Production Engineering, University of Naples Federico II, Piazzale Tecchio 80, Naples, Italy

Abstract. Powder bed additive manufacturing allows for the production of fully customizable parts and is of great interest for industrial applications. However, the repeatability of the parts and the uniformity of the mechanical properties are still an issue. More specifically, the physical mechanism of the spreading process of the powders, which significantly affects the characteristics of the final part, is not completely understood. In powder bed fusion technologies, the spreading is performed by a device, typically a roller or a blade, that collects the powders from the feedstock and successively deposits them in a layer of several dozens of microns that is then processed with a laser beam. In this work, an experimental approach is developed and employed to study the powder spreading process and analyze in detail the motion of the powders from the accumulation zone to the deposition stage. The presented experiments are carried out on a home-made device that reproduces the spreading process and enables the measurement of the characteristics of the powder bed. Furthermore, the correlation with the process parameters, e.g., the speed of the spreading device, is also investigated. These results can be used to obtain useful insights on the optimal window for the process parameters.

Keywords. Powder Bed, Additive Manufacturing, Experimental, Laser Powder Bed Fusion

\section{Introduction}

In the latest years Laser Powder Bed Fusion (LPBF) has affirmed as the go to additive technology for the production of parts for critical applications in fields such as the aerospace and biotech industry [1]. It enables the production of fully customizable parts with respect to both the geometrical features and the mechanical properties. Despite the indisputable advantages listed before, some issues still prevent the additive manufacturing from expressing its full potential as a revolutionary manufacturing technology. More specifically, the repeatability of the parts and the uniformity of the mechanical properties throughout the printing chamber are still not fully guaranteed and defects due to a non-optimal selection of the parameters are still not completely addressed [2]. The aforementioned issues can result in a diminished technological and economic reliability of the process. In the authors opinion the root cause of all the described issues can be found in the often overlooked first stage of the printing process: the spreading and deposition of the powder bed. In fact, a powder bed with uneven characteristics (e.g., packing factor, powder size distribution, effective layer thickness) throughout its area makes it impossible to select the optimal value of the laser parameters (i.e., hatch spacing, power and so on) for the entirety of the printing chamber. At the same time, a deeper understanding of the influence of the spreading parameters (i.e., recoating device's speed, layer thickness, the total quantity of powder to be spread for each layer) on the state of the powder bed can lead to a proper selection of the parameters in order to attain overall better characteristics of the finished part. 
There are a great number of studies on the influence of the laser parameters on the mechanical properties of the printed parts [3], but very few focus on the study of the powder bed in its loose state. Some studies [4] [5] use a numerical approach to identify the influence of the spreading parameters, however such an approach, whilst being fundamental to identify the spreading mechanism on a particle scale cannot offer an accurate modelling of the process in its full scale. Therefore, in order to properly identify the influence of the spreading parameters an experimental analysis is needed. Presently, a complete procedure for the experimental investigation of the powder bed in its loose state is still lacking, Ali et al. [6] proposed an approach based on the use of a UV cured polymer to "freeze" the particles and perform successive ex-situ analysis, Liu et al. [7] designed a container and printed it to measure and analyze the powders trapped inside it. The described approaches, while giving insight on some aspects of the process still have some limitations. It is impossible to determine a priori the volume of powders trapped by the polymer, and that the addition of the polymer will not cause a rearrangement of the deposited powder. At the same time, the presence of the printed part will probably influence the spreading process. Some researchers [8] [9] focused their attention on the in-situ monitoring of the spreading process via image analysis during the printing, which enables to monitor faulty operating conditions and the formation of defects but fails to fully isolate the influence of the spreading parameters.

In order to address the described issues, the authors propose a more organic, direct and faster experimental approach aimed at identifying the key characteristics of the powder bed and to correlate it to process parameters and to the zone of the powder bed investigated. A deeper understanding of the spreading process is of key industrial interest since a slight modification in the layer thickness or the spreading speed can dramatically affect the productivity of the printing. More specifically, being able to select a faster combination without sacrificing the quality of the powder bed and hence of the final manufact.

\section{Materials and methods}

\subsection{The powders}

The powder selected for the experimental investigation is aluminum, a common material for additive manufacturing applications. The powders were characterized via microscope analysis. An image obtained via a Hitachi TM 3000 tabletop scanning electron microscope (SEM) of the powders is presented in Fig. 1. It can be noted that the selected powders have a low level of sphericity and a rather broad dimension range. These characteristics are typical of recycled powders, powders that have been used in several 3D printing jobs [10]. In fact, due to the high price of said powders, the reuse is mandatory to avoid the skyrocketing of the process' cost. These powders will presumably hinder the spreading process to some extent. In this way, a reference for the attainable characteristics of the powder bed will be set, for future comparison. 


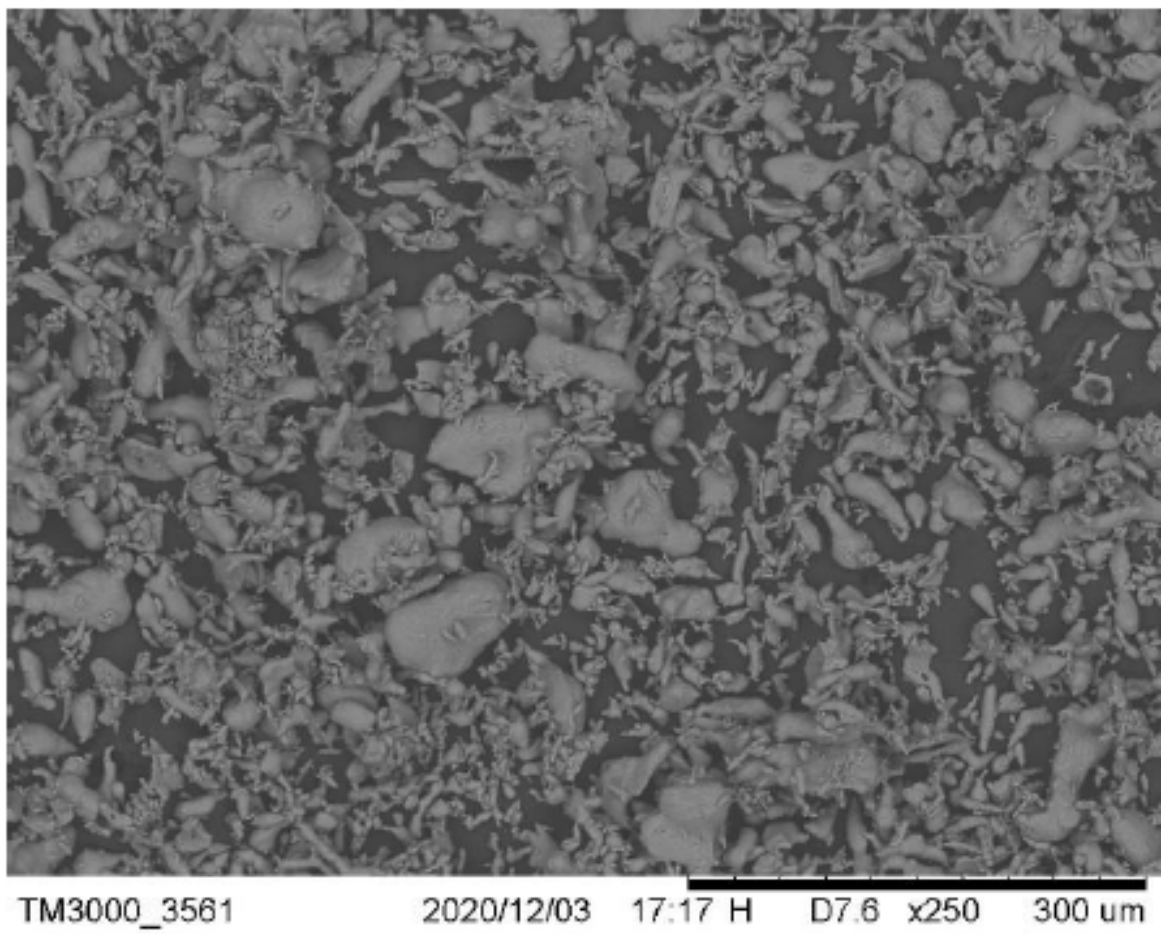

Fig. 1 SEM image of the powders (250X)

\subsection{The experimental device}

The experimental analysis of the powder bed that will be presented in the next sections were carried out on a purposely designed experimental device. The device, presented in Fig. 2 faithfully reproduces the powder spreading module of an actual 3D printer, namely a Concept laser M2 cusing based on the laser powder bed fusion technology in order to guarantee that the spreading conditions are the same as a real printing process. It consists of two plates: one where the powders are deposited in a thin layer to be processed by the laser source and one for the feedstock of powders that supply new material for the successive layers. Both of those two plates can be adjusted independently via a pair of micrometric screws, enabling to vary the ratio between the quantity of powder required for the layer and powder provided by the feedstock. The spreading itself is performed by a recoater, in this case a vertical blade made of a soft rubber material, whose speed can be adjusted in the range between $100 \mathrm{~mm} / \mathrm{s}$ and $10 \mathrm{~mm} / \mathrm{s}$. Said range was deemed sufficient to analyze the effects of the recoating device speed on the spreading process because higher velocities are not standardly used in the real 3D printer the device was derived from while velocities lower than $10 \mathrm{~mm} / \mathrm{s}$ are of no industrial interest since the overall duration of the process would be negatively affected.

The temperature of the building plate is monitored and regulated in order to minimize the impact of the external conditions. The whole apparatus is placed in a closed chamber in order to isolate the device from the external environment and for safety reasons. The operating conditions of the device can be monitored via a LED display. As mentioned before, the powder bed can be characterized by several features. The characteristics chosen in this work are the density of the powder bed and its surface morphology. In the next sections the experimental approach used to obtain said characteristics will be discussed. 


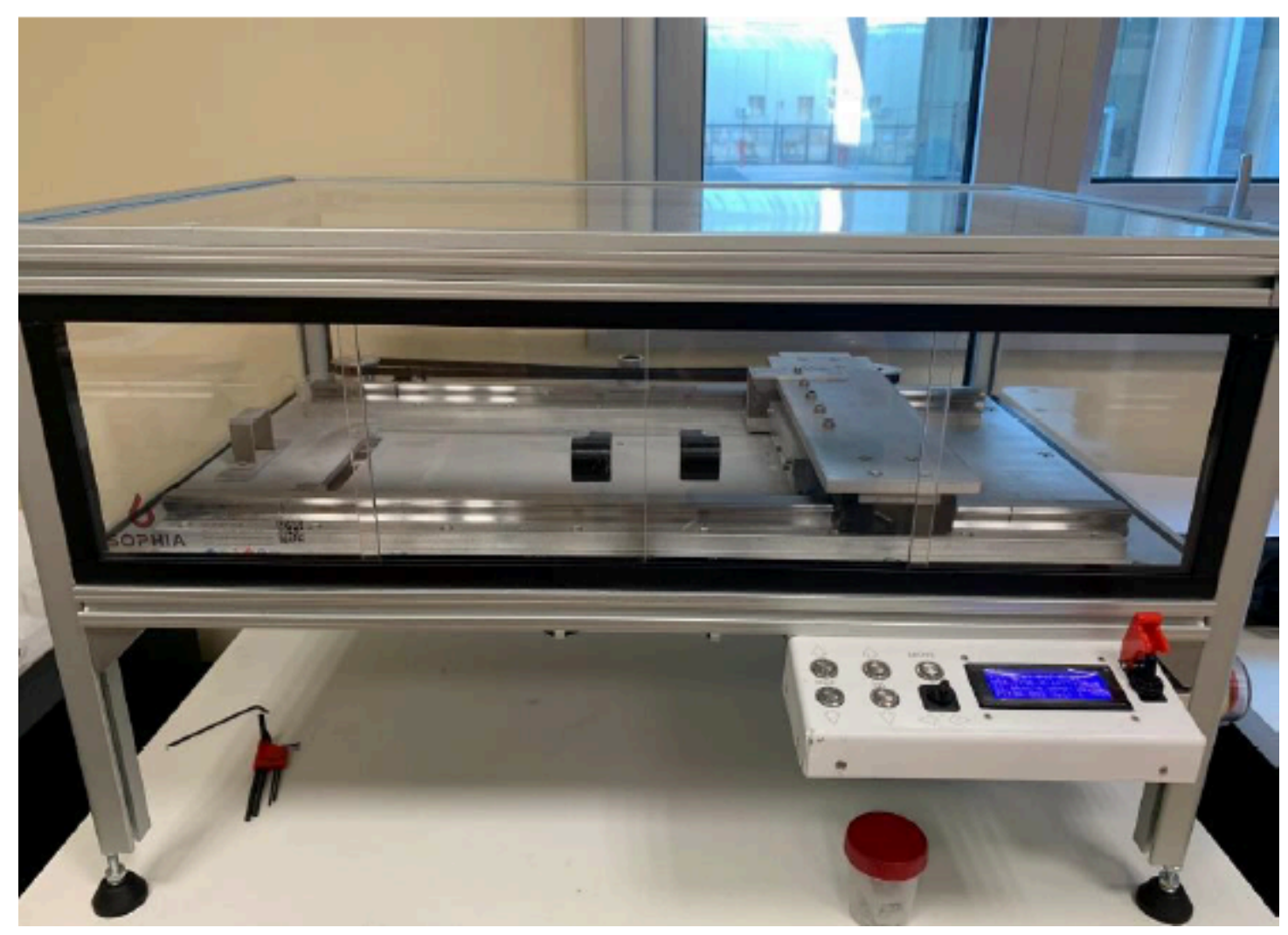

Fig. 2 Experimental spreading device

\subsection{Packing factor}

In order to identify the packing factor of the powder bed in the location of interest a sampling device (presented in Fig. 3) was specifically designed for the sampling of the shallow powder bed consisting of only 15 layers. The described device was carefully weighted with a precision balance before and after the sampling and was carefully cleaned between each sampling to remove any leftover powders. 
a)

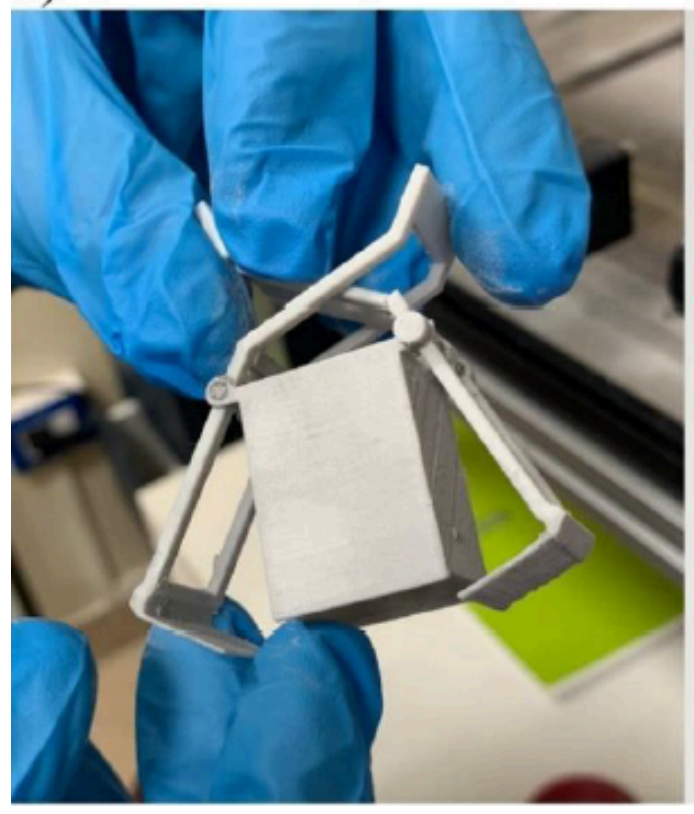

b)

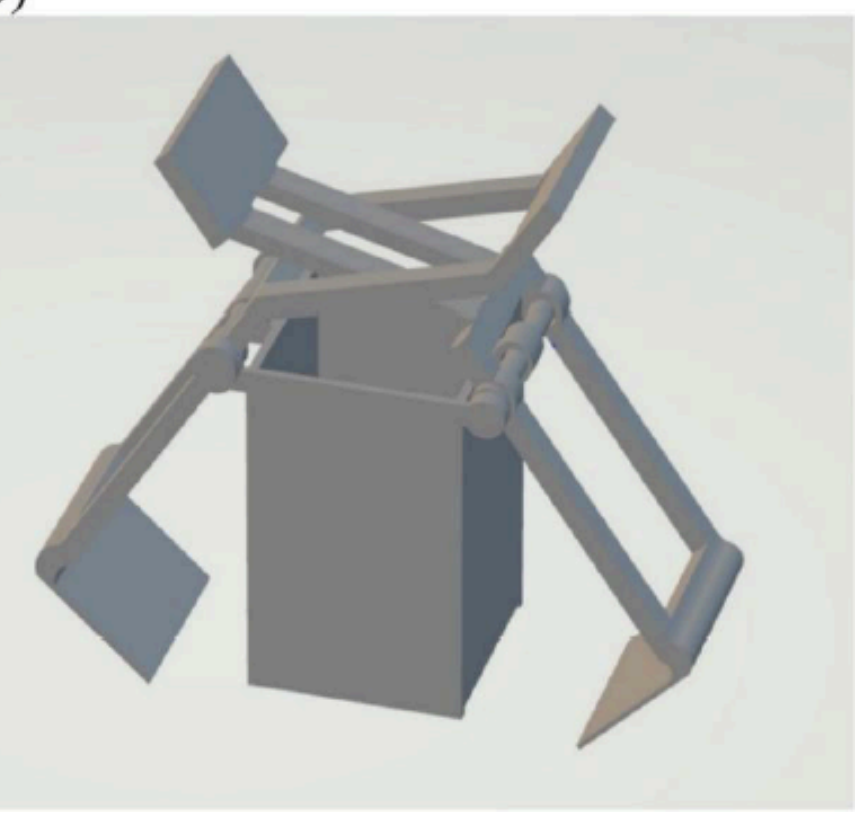

Fig. 3 Sampling device (a) and its 3D design (b)

\subsection{Layer morphology}

Having a layer of powders with a strongly discontinuous surface is not advisable since it can disturb the interaction with the laser during the printing process and implies that the mass of powder is strongly in-homogeneously distributed. In order to identify and obtain a quantitative measurement of this parameter the following procedure was followed: using a Wi-Fi microscope (MICFIT6) and a custom support an in-situ snapshot of the zone of interest (a $1 \mathrm{~mm} \times 1 \mathrm{~mm}$ square) of the powder bed was captured as shown in Fig. 4. A relatively small area was chosen for the acquisition in order to deny to some extent higher-order disturbances of the powder bed. The choice of the Wi-Fi microscope was justified from the possibility to investigate the powder bed in situ and without presumably altering the bed itself. The so obtained images were then processed via an image analysis software (ImageJ) following an approach similar to that of Than Puc et al. [11] the out area out of focus (and hence out of the intended deposition plane) was identified using an edge detection algorithm and quantified. 


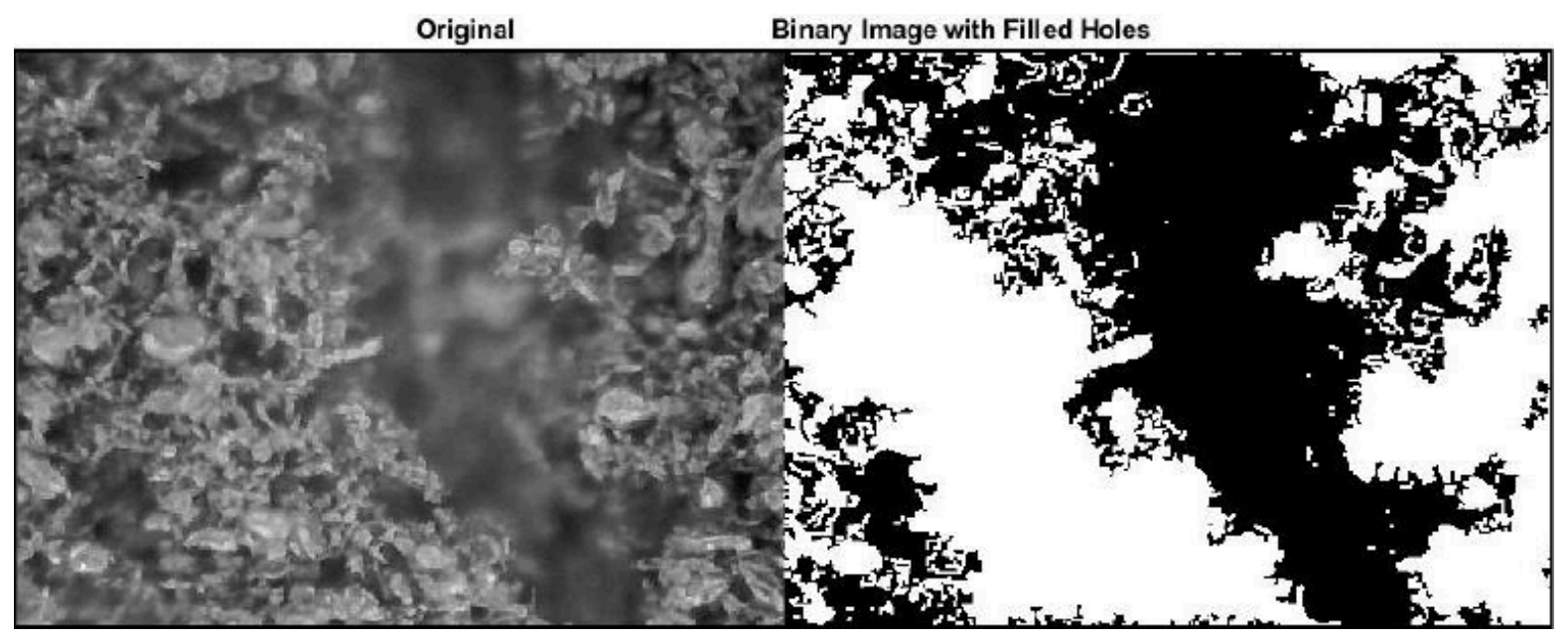

Fig. 4 Snapshot of a zone of the powder bed and the result of the post processing

\subsection{Experimental plane}

The experimental approach described above was used to identify the effect of the variation of the spreading process on the quality of the powder bed. A simple DoE was set up to investigate the effect of the variation of the layer thickness and of the spreading device's speed. More specifically, the selected levels for the layer thickness were: $30 \mu \mathrm{m}, 50 \mu \mathrm{m}$ and $100 \mu \mathrm{m}$. What drove the choice of the layer thickness values was the mean and bigger diameter of the particles. In fact, there are no particles bigger than $100 \mu \mathrm{m}$ and therefore with such a layer thickness value no jamming due to the mechanical blocking of the space between the blade and the existing layer is to be expected, while for the $50 \mu \mathrm{m}$ and $30 \mu \mathrm{m}$ values the fraction of particles that can potentially give rise to the described jamming phenomenon increases significantly. In this way the interaction between the particles' dimension and the overall effect of the layer thickness can be enlightened. As for the recoating device's speed the selected level were: $60 \mathrm{~mm} / \mathrm{s} 30 \mathrm{~mm} / \mathrm{s}$ and $10 \mathrm{~mm} / \mathrm{s} .60$ $\mathrm{mm} / \mathrm{s}$ can be considered a standard speed for 3D printing applications while the other levels are fairly slower than those used in industrial applications. More specifically, the $10 \mathrm{~mm} / \mathrm{s}$ level is practically of no industrial interest due to the negative impact on the duration of the printing process but was taken into consideration to better understand the principles regulating the process. A summary of the combination of parameters used for the experiments is listed in Table 1. In order to guarantee repeatability and to identify any variation in the powder bed along the spreading direction, for each of the combinations the powder bed characteristics will be evaluated at four distinct locations, namely the four corners of the building plate as shown in Fig. 5. 


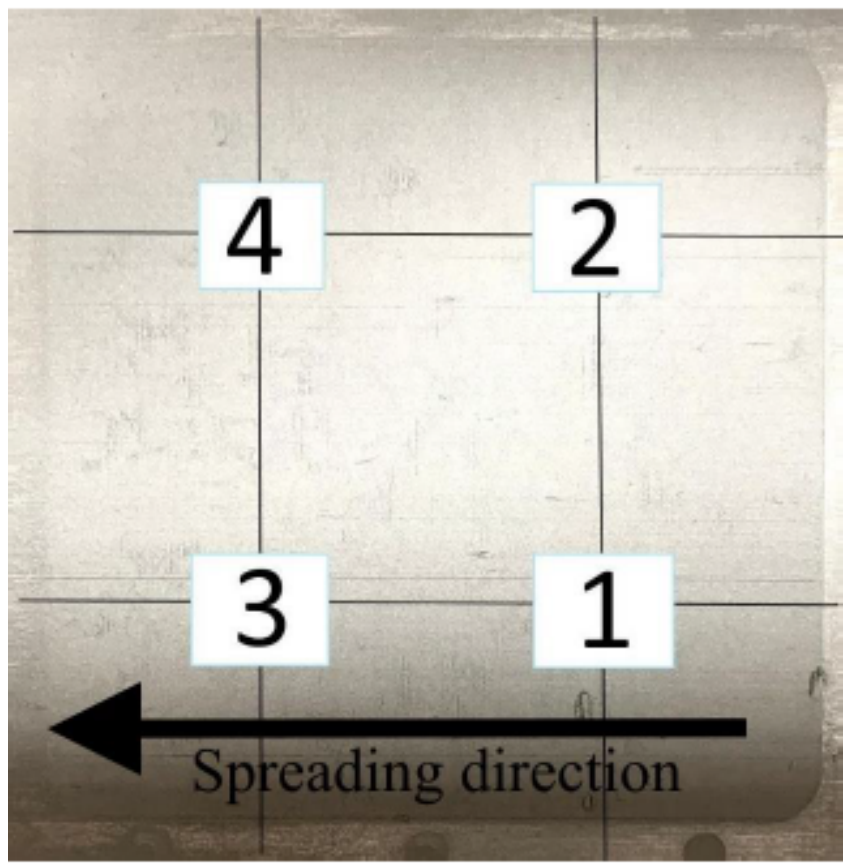

Fig. 5 Investigated zones on the deposition plate

Table 1. Parameters of the experiments

\begin{tabular}{ccc}
\hline Experiment & $\begin{array}{c}\text { Layer } \\
\text { thickness [ } \mu \mathrm{m}]\end{array}$ & $\begin{array}{c}\text { Recoater's } \\
\text { speed } \\
{[\mathrm{mm} / \mathrm{s}]}\end{array}$ \\
\hline 1 & 100 & 10 \\
\hline 2 & 100 & 30 \\
\hline 3 & 100 & 60 \\
\hline 4 & 50 & 30 \\
\hline 5 & 50 & 60 \\
\hline 6 & 30 & 30 \\
\hline 7 & 30 & 60 \\
\hline
\end{tabular}

\section{Results and discussion}

The next section will cover the results of the above-described experiments. More specifically, the attention will be focused on the influence of the process parameters on the powder bed characteristics and on highlighting the identified spreading mechanism. Fig. 6 shows the variation of the relative density (normalized with respect to that of bulk aluminum) for different spreading speeds at a fixed layer for the "front" and "back" zones of the powder bed (corresponding respectively to zone 1-2 and 3-4 in Fig. 5). It can be observed that a slower spreading speed appears beneficial for a layer value of $100 \mu \mathrm{m}$ while for a layer value of $50 \mu \mathrm{m}$ and $30 \mu \mathrm{m}$ a decreasing spreading speed negatively affects the density of the powder bed. In the author's opinion the reason for the dependence can be found in 
the ratio between the layer thickness and the powder's maximum dimension. In fact, as can be seen from Fig. 1 there are basically no particles bigger than $100 \mu \mathrm{m}$ and therefore for that value of the layer said ratio can be considered equal to 1 while for layers of $50 \mu \mathrm{m}$ and $30 \mu \mathrm{m}$ it surely is less than 1 . In fact, for the last case the bigger particles can act as a cap, blocking the space between the blade and the already deposited layer and can benefit from the more dynamic action of a fast spreading speed, while for a sufficiently thick layer the spreading speed promotes a more efficient deposition [12]. The change in the spreading mechanism is also confirmed by an observed shift in the forming mechanism of the powder layer: for the lower values of the thickness the powder heap accumulated in front of the blade only started to significantly decrease in the final zone of the deposition plate, while for the higher thickness value it deceased monotonically. This hypothesis will be verified in upcoming works via a more specific set of experiments and with a numerical approach, but it was worthwhile to point it out. Moreover, it appears that a thicker layer makes it possible to achieve a denser powder bed. This further hints at a filtering effect of a smaller gap [13]. Anyhow, it should be noted that a powder bed with an overall lower but more stable may be preferable compared to a powder bed with higher peaks of density but more unevenly distributed. That appears to be the case in Fig. 6 (a) for increasing speed levels.

a)

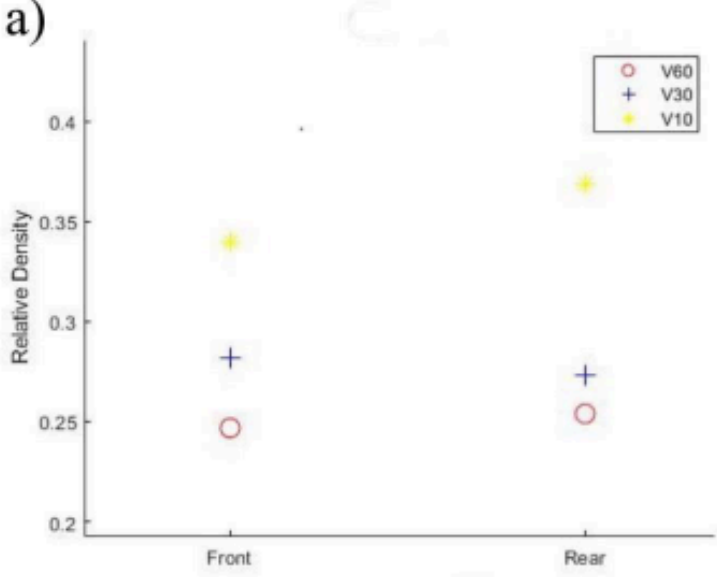

c) b)

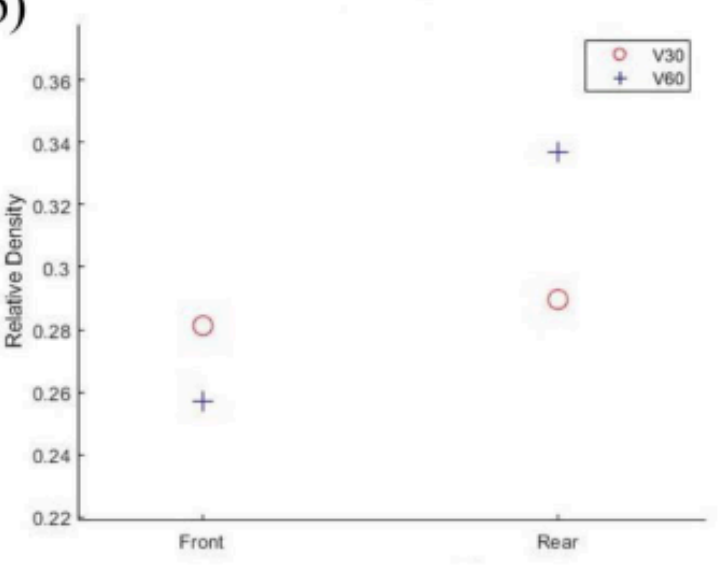

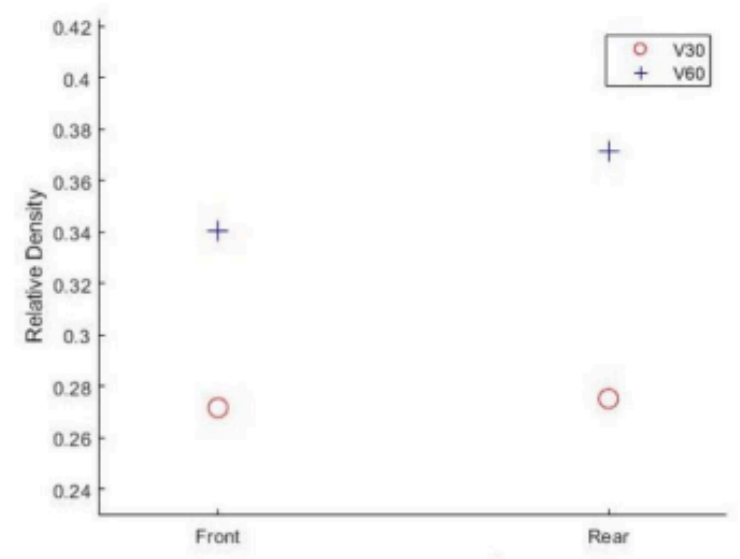

Fig. 6 Relative density of the powder bed at "front" and "rear" locations for a layer of $100 \mu \mathrm{m}$ (a), $50 \mu \mathrm{m}$ (b) and $30 \mu \mathrm{m}$ (c)

For what concerns the morphology of the deposited layer, the results are plotted in Fig. 7. While little to none influence 
of the process parameters emerges, contrary to what can be grasped from the literature $[12,14]$, it is interesting to note that for all the combinations of parameters the value of the in-focus zone has an average value that corresponds to roughly half of the total area taken into consideration, and the out of focus zones are rather extensive, meaning that the powder bed has a rather rough surface, with discontinuities and surface patterns that extend way beyond the typical dimensions of the powders. This lack of dependence can be due to the high aspect ratio of the particles since it can give rise to phenomena of mechanical interlocking between the particles that can affect the surface roughness.

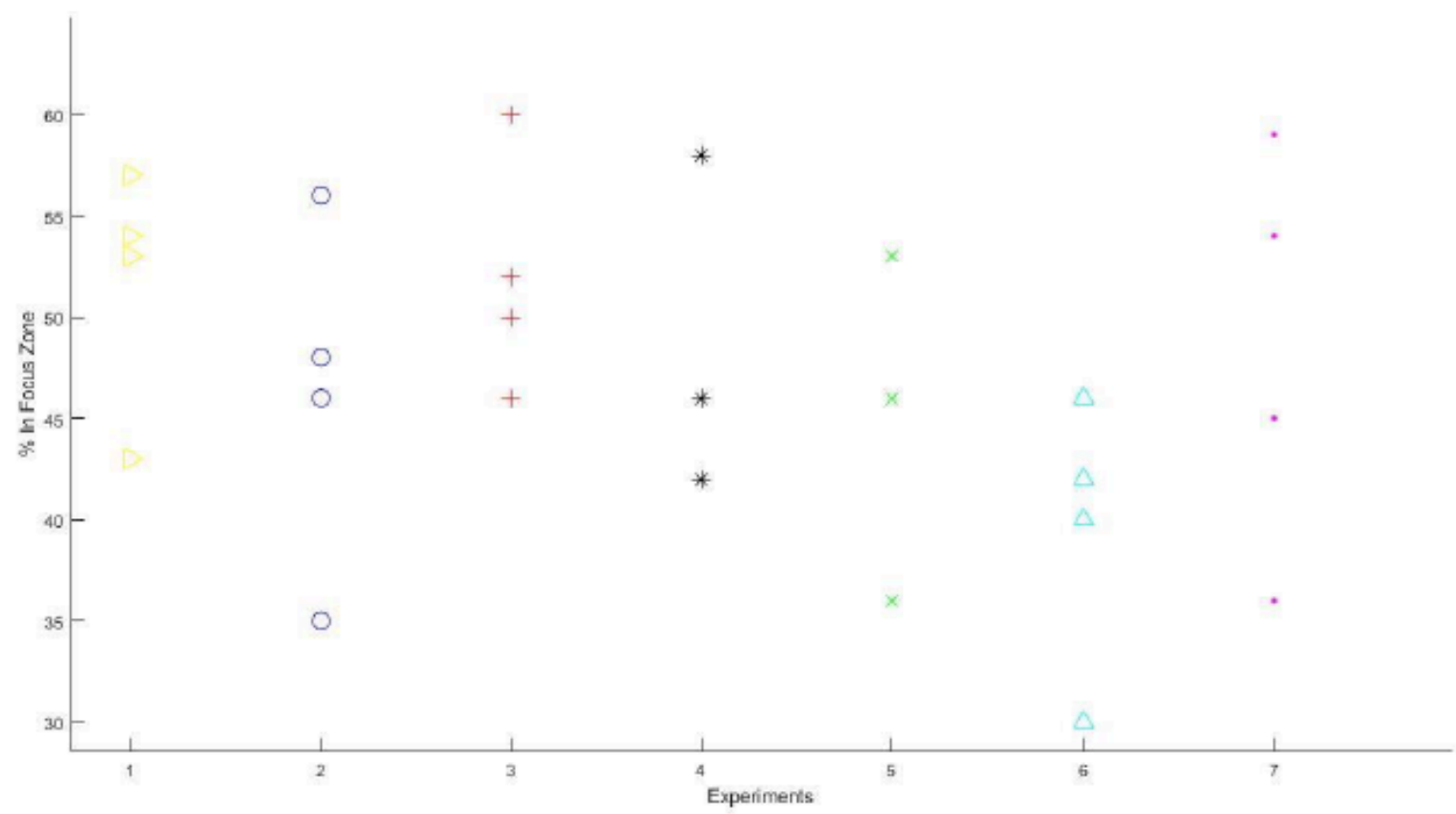

Fig. 7 Fraction of the in-focus zone for the set of experiments

\section{Conclusions}

In this work an experimental approach for the characterization of the powder bed deposited in laser powder bed fusion (LPBF) technologies was presented and used to investigate the influence of the process parameters on said characteristics. The experimental approach proved to be valid in giving insights into the spreading process. The main findings can be resumed as follows:

- A thicker layer promotes the deposition process and results in a denser powder bed.

- The recoating speed appears to have a beneficial effect for high values of the layer thickness, while it has a detrimental effect for low values of the layer thickness.

- The surface of the deposited layer appears to be rather insensitive to the process parameters.

However, since said dependences appear to be related to the powder size and morphology, other experimental campaigns with more control over these aspects and an extension of the experimental approach to include the analysis of the variation of the particle size distribution (PSD) seem appropriate to verify the hypothesis here formulated.

\section{Acknowledgements}

The authors would like to acknowledge Sophia High Tech for developing the experimental equipment and supporting all the activities. 


\section{Bibliography}

[1] Frazier, WE. Metal additive manufacturing: A review. Journal of Materials Engineering and Performance, 2014, 23, 1917-28.

[2] Gong, H. Rafi, K. Gu, H. Starr, T. Stucker, B. Analysis of defect generation in Ti-6Al-4V parts made using powder bed fusion additive manufacturing processes. Additive Manufacturing, 2014, 1, 87-98.

[3] Metel, A. Stebulyanin, M. Fedorov, S. Okunkova, A. Power Density Distribution for Laser Additive Manufacturing (SLM): Potential, Fundamentals and Advanced Applications. Technologies, 2018, 7, 5.

[4] Chen, H. Wei, Q. Zhang, Y. Chen, F. Shi, Y. Yan, W. Powder-spreading mechanisms in powder-bed-based additive manufacturing: Experiments and computational modeling. Acta Materialia, 2019, 179, 158-71.

[5] Fouda, YM. Bayly, AE. A DEM study of powder spreading in additive layer manufacturing. Granular Matter, 2020, 22.

[6] Ali, U. Mahmoodkhani, Y. Imani Shahabad, S. Esmaeilizadeh, R. Liravi, F. Sheydaeian, E. Huang, KY. Marzbanrad, E. Vlasea, M. Toyserkani, E. On the measurement of relative powder-bed compaction density in powder-bed additive manufacturing processes. Materials and Design, 2018, 155, 495-501.

[7] Liu, B. Wildman, R. Tuck, C. Ashcroft, I. Hague, R. Investigaztion the effect of particle size distribution on processing parameters optimisation in selective laser melting process. 22nd Annu. Int. Solid Free. Fabr. Symp. - An Addit. Manuf. Conf. SFF 2011, 2011, p. 227-38.

[8] Liu, Y. Blunt, L. Zhang, Z. Rahman, HA. Gao, F. Jiang, X. In-situ areal inspection of powder bed for electron beam fusion system based on fringe projection profilometry. Additive Manufacturing, 2020, 31.

[9] Kalms, M. Narita, R. Thomy, C. Vollertsen, F. Bergmann, RB. New approach to evaluate 3D laser printed parts in powder bed fusion-based additive manufacturing in-line within closed space. Additive Manufacturing, 2019.

[10] Slotwinski, JA. Garboczi, EJ. Stutzman, PE. Ferraris, CF. Watson, SS. Peltz, MA. Characterization of metal powders used for additive manufacturing. Journal of Research of the National Institute of Standards and Technology, 2014, 119, 460-93.

[11] Tan Phuc, L. Seita, M. A high-resolution and large field-of-view scanner for in-line characterization of powder bed defects during additive manufacturing. Materials and Design, 2019, 164.

[12] Parteli, EJR. Pöschel, T. Particle-based simulation of powder application in additive manufacturing. Powder Technology, 2016, 288, 96-102.

[13] Zhao, Y. Koizumi, Y. Aoyagi, K. Yamanaka, K. Chiba, A. Characterization of powder bed generation in electron beam additive manufacturing by discrete element method (DEM). Mater. Today Proc., 2017.

[14] Haeri, S. Wang, Y. Ghita, O. Sun, J. Discrete element simulation and experimental study of powder spreading process in additive manufacturing. Powder Technology, 2017, 306, 45-54.

PDF automatically generated on 2021-05-20 06:27:56

Article url: https://popups.uliege.be/esaform21/index.php?id=2433

published by ULiège Library in Open Access under the terms and conditions of the CC-BY License

(https://creativecommons.org/licenses/by/4.0) 